\title{
FOREWORD
}

\section{Special issue on musical acoustics}

\author{
Shigeru Yoshikawa \\ Department of Acoustical Design, Kyushu Institute of Design, \\ 4-9-1 Shiobaru, Minami-ku, Fukuoka, 815-8540 Japan
}

Musical acoustics research in Japan can be characterized by its diversity. The scope of its interests, approaches, and techniques has been broadened through active joint meetings with the Technical Committee (TC) on Music and Information Science of the Information Processing Society of Japan, the TC on Speech of the Institute of Electronics, Information and Communication Engineers, and the Japanese Society for Music Perception and Cognition. Of course, traditional topics on musical instruments and their tones as well as other topics on music performance, computer music, etc. have been discussed at almost monthly meeting of the TC on Musical Acoustics of our ASJ.

These varied and vigorous activities in musical acoustics prompted the decision that the time was ripe for a special issue of Acoustical Science and Technology, an enhanced version of the Journal of the Acoustical Society of Japan (E). No particular theme was set in order to provide a common ground for the contribution of results from interdisciplinary researches. It was also hoped that this special issue would promote scientific exchange more effectively in the internationalizing community by publishing our state of the art in musical acoustics at the beginning of 21 st century.

The papers received were subjected to the normal process of peer review, and the result is the present volume. Several foreign experts were involved in the review process to ensure international standards. This was the first such attempt in our Journal. There are seven contributed papers and two invited reviews. The contributed papers consists of three papers on musical instruments (the Chikuzen biwa, Sanukite stone-made hokyo, and the flute), three papers on musical perception and performance concerning the rhythm or pure tones, and one paper on the processing of the music score in the relation to music performance.
Two invited reviews focus on recent progress in musical instrument acoustics. The interaction between nonlinear physics and musical acoustics is clearly recognized there. Also, various technologies have been applied to make precise measurement and overall visualization, from which insights on nonlinear dynamics and the sophistication of the physical models are provided. These two reviews, as well as the special issue on musical wind instrument acoustics in Acustica-Acta Acustica (Vol. 86, July/August 2000), seem to mark a milestone in 20th-century musical instrument acoustics and suggest the direction of musical instrument acoustics for the 21st century.

A relevant combination of our scientific studies with the practice of instrument makers, the performance of music, and the perception of musicians will promote the maturity and the influence of musical acoustics in our human society. Links and connections with our human society and environment should be crucial for the future development of musical acoustics. Our goal will be attained by the enlargement of research viewpoint, the reinforcement of in-house research activity, and every endeavor for international and public appreciation of Japanese musical acoustics.

I would like to express my sincere acknowledgments for proper comments and helpful suggestions from all the referees who contributed their time and expertise in the preparation of this special issue. Also, I would like to deeply thank Prof. Neville H. Fletcher of Australian National University and Prof. Thomas D. Rossing of Northern Illinois University for their contribution of invited reviews and long-term support to this special issue from the very beginning of its planning stage. Finally, I am very grateful for the valuable work of my fellow Associate Editor for Musical Acoustics on the Editorial Board, Naotoshi Osaka. 